\title{
Approaches to testing vehicle passive safety based on a virtual test bench and a digital twin
}

\author{
Dmitry Bogdanov ${ }^{1}$, Anton Zhilenkov ${ }^{2, *}$, and Elena Zhilenkova ${ }^{1}$ \\ ${ }^{1}$ Peter the Great St. Petersburg Polytechnic University, 195251, St. Petersburg, Russia \\ ${ }^{2}$ Saint Petersburg State Marine Technical University, 190121, St. Petersburg, Russia
}

\begin{abstract}
The article presents the results of the implementation of work in the field of creating virtual test benches for testing vehicle passive safety in the event of an impact. A number of results obtained on the basis of implemented virtual platforms and benches are given. The advanced technologies in the field of creating virtual benches and digital twins are also shown. The illustrations on the problems of testing A-pillars and the vehicle roof, including the cases of a frontal impact are given. Certain considerations are given on the general concept of digital design based on digital twins and virtual test benches. The critical impact on the adequacy of the digital twin and the benches and platforms built on its basis is highlighted. The accuracy and convergence of the mathematical model of the designed product and the physical processes occurring in it, the used numerical methods of computer simulation, finite element models and their optimization is pointed out.
\end{abstract}

\section{Introduction}

One of the most important tests for a designed vehicle is a crash test. Specifically, it is an assessment of the passive safety of passengers and the driver in a frontal, rear or side impact, as well as during a vehicle rollover.

The success of scientists and engineers in the field of creating virtual benches drew attention to the problems of assessing the passive safety of the designed vehicles. Below are the results obtained on the designed virtual platform, which is built on the basis of the digital twin of a vehicle. This approach allows the assessment and optimization of a number of parameters to be realized at a new level.

Working with the digital twin of the designed product provides an opportunity to consider the smallest details, which at the same time have a significant impact on the level of compliance with the requirements of international standards for vehicle safety, etc.

\section{Virtual benches for testing vehicle passive safety}

In the development of a digital platform for the design and testing of products created, particular attention needs to be given to testing issues. Namely, whether the product created

\footnotetext{
*Corresponding author: zhilenkovanton@gmail.com
} 
meets the requirements for operational safety. Such a complex technical product as a vehicle today must be equipped with active systems to ensure the safety of the driver and passengers.

However, the main requirement is compliance with safety regulations, in particular with those established by the Economic Commission for Europe of the United Nations (Regulation No. 29). These rules regulate the requirements for the vehicle passive safety. To implement testing of the designed vehicle for compliance with these rules, virtual benches were developed to assess passive safety indicators in the following cases: in frontal, side, rear impacts and during vehicle rollover.

For the implementation of such benches, first of all, sufficiently accurate mathematical models are needed [1-3]. These models should reflect the physical processes occurring during the creation of the testing environment and their computer implementations. Such models form the so-called digital twin of the product. The accuracy of the model makes it possible to obtain fairly accurate assessments, i.e. the results of testing carried out on a digital twin based on a virtual bench.

One of the basic models required for assessing vehicle passive safety in various situations and under various conditions is the model of the vehicle stiffness and strength. Figure 1 shows a visualization of the assessment of the vehicle stiffness and strength, obtained on the basis of the analysis and optimization of the digital twin.

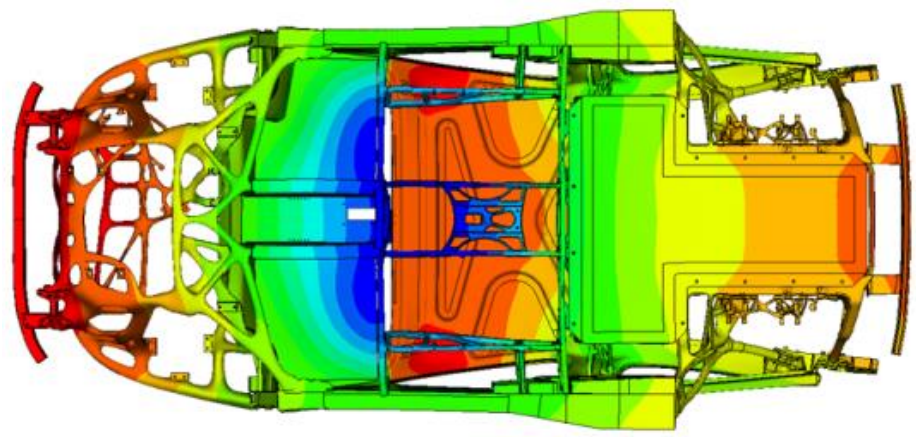

Fig. 1. The results of the analysis of the stiffness and strength of the vehicle body, obtained on a virtual bench.

A general view of the digital model of the designed product, which can be visualized by the digital platform software, is shown in Figure 2.
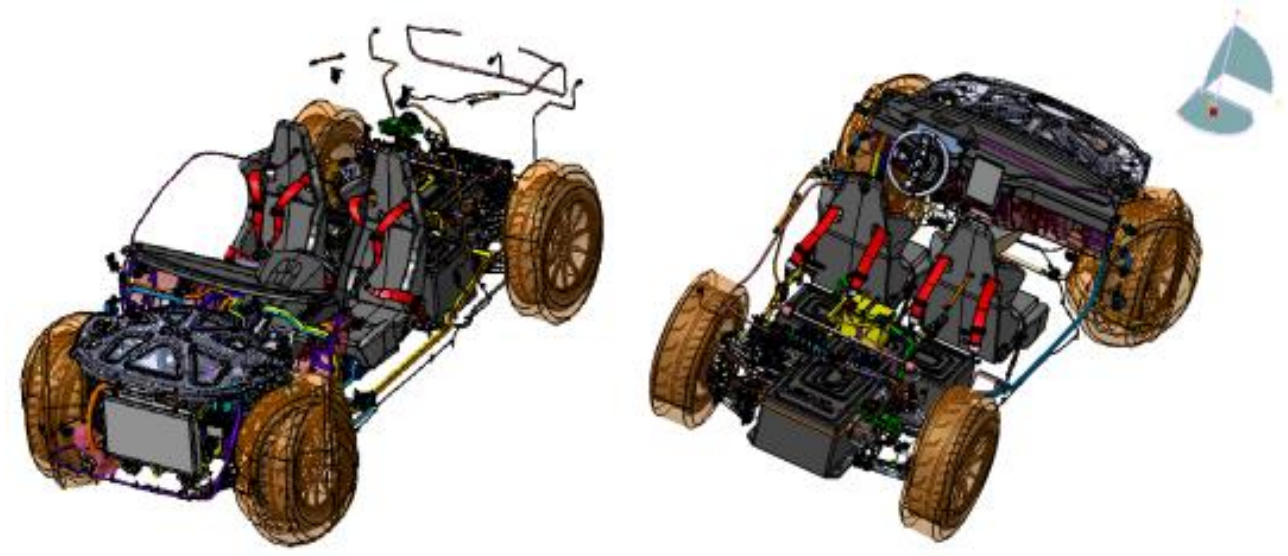

Fig. 2. Model of a vehicle designed on the basis of a digital platform. 


\section{Assessment of the passive safety of the digital twin in frontal impact}

The digital model allows you to visualize the assembled product, as well as its individual elements and systems [4-5]. To assess vehicle passive safety, the body, pillars, etc. can be separately displayed on the screen.

Figure 3 shows a visualization of tests on a virtual bench to assess passive safety in the event of a frontal impact.

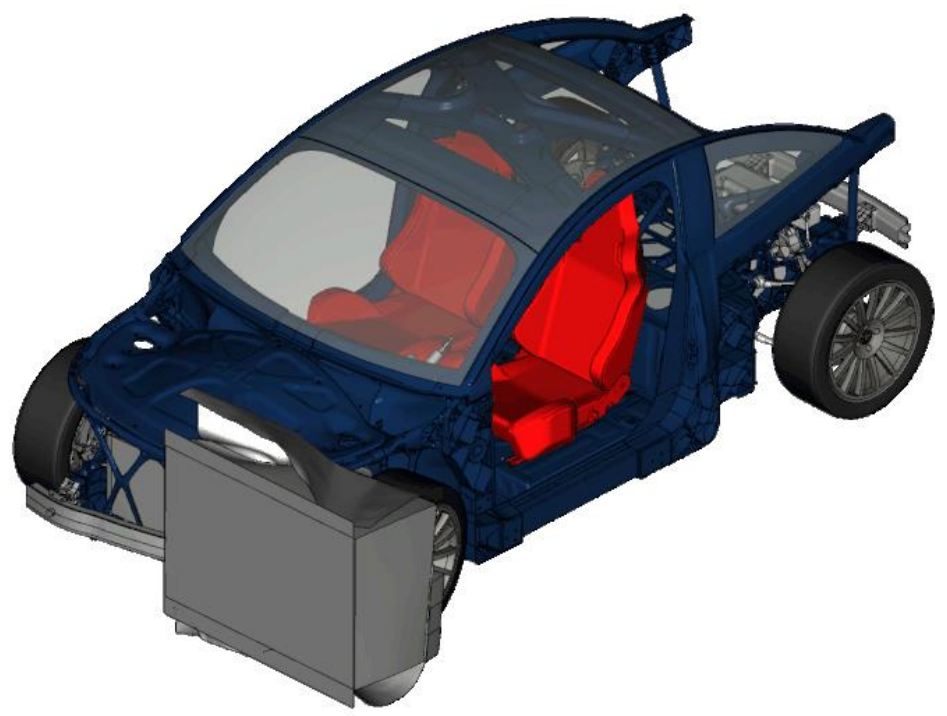

Fig. 3. Assessment of the passive safety of a digital twin of a vehicle on a virtual bench when simulating a frontal impact.

The user conducting the assessment can explore the processes taking place in the system under test, visualizing it in various projections and highlighting the process details and structural elements of interest to him (Fig. 4).

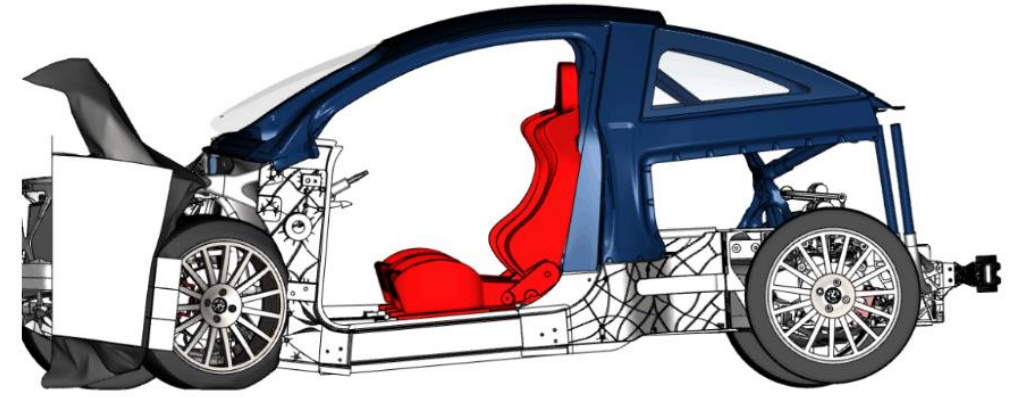

Fig. 4. Study of the influence of a frontal impact on a digital twin of a vehicle.

\section{Assessment of the passive safety of the digital twin in frontal impact}

The developed digital platform allows visualizing the process and assessing passive safety in the event of a side impact of a designed vehicle $[6,7]$. 


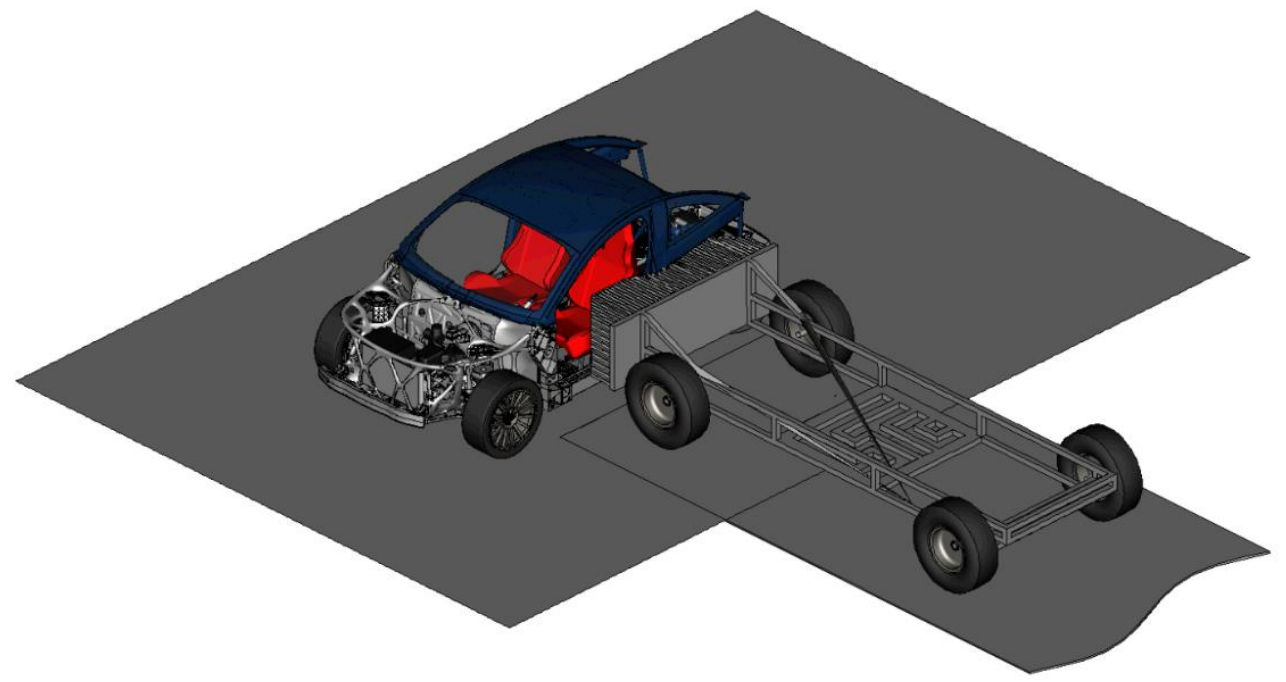

Fig. 5. Simulation of a side impact carried out on a virtual bench of a digital platform.

A similar approach to assess and optimize the safety based on a computer model is used.

\section{Assessment of the passive safety of the digital twin in frontal impact}

The examples of similar assessments performed on virtual test benches for commercial vehicles are further described. Figure 6 shows the result of visualization of the crash test subroutine interface when simulating a frontal impact of a commercial vehicle product.

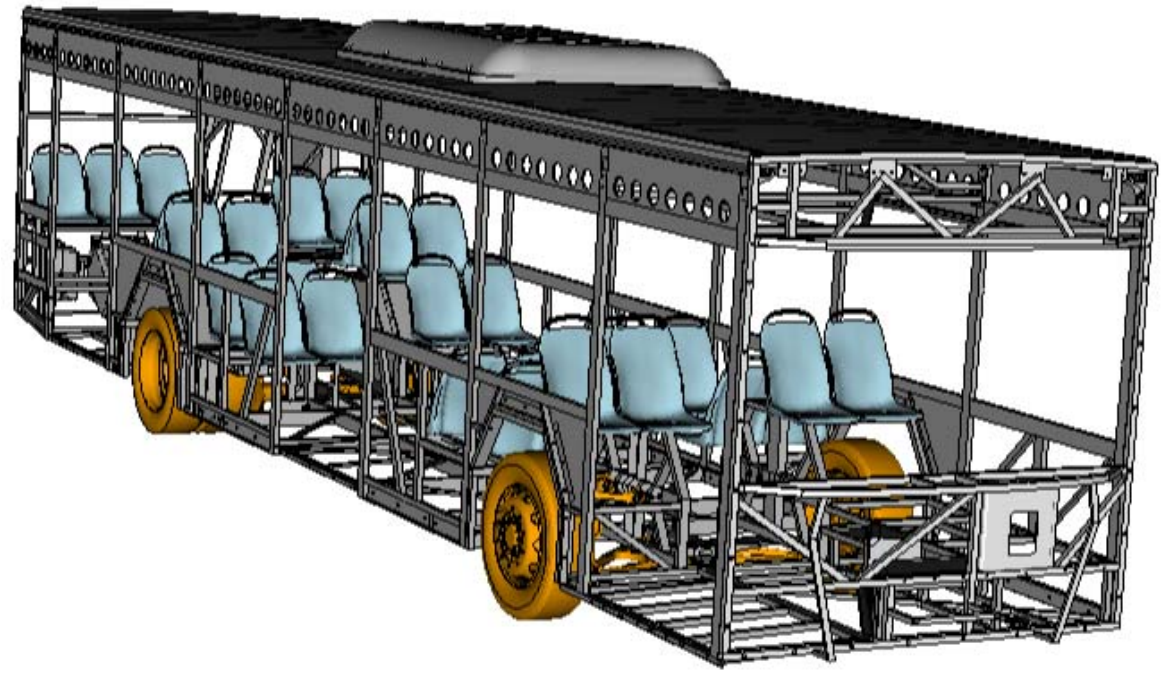

Fig. 6. Assessment of passive safety in frontal impact.

The developed program allows visualizing and assessing the stress-strain state of the vehicle cab and also building a map of stress distribution in the body, etc. 
Figure 7 shows the results obtained also for the case of a frontal impact, but on the Apillars of the vehicle [8]. As it can be seen, the user can get a detailed understanding of the influence of the impact results on any element of the vehicle body.

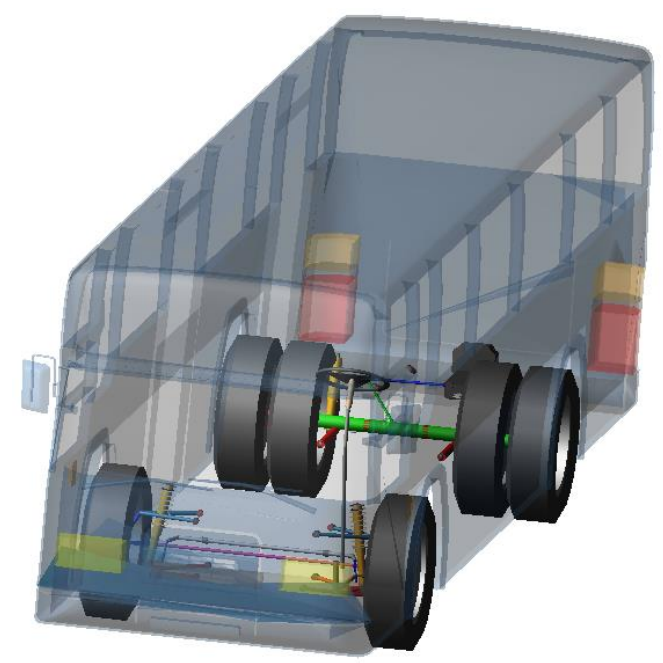

Fig. 7. Assessment of passive safety in case of a frontal impact of vehicle pillars on a virtual bench.

Similarly, a crash test is implemented with a simulation of the case of a rear impact. If there is no possibility of testing digital twins, all such tests have to be carried out on physical models (Fig. 8). However, it is much more expensive and time-consuming.

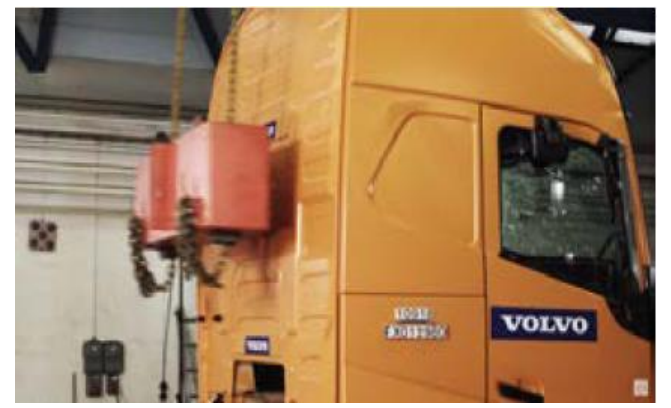

Fig. 8. Passive safety assessment on a physical model with simulation of a rear body impact.

Testing on simulation models, digital twins, virtual benches and digital platforms not just makes the development and verification of designed products cheaper [9]. It makes it possible, without making a physical prototype, to assess those parameters and processes that cannot be carried out on a physical model. It also provides an opportunity to assess processes in which difficulties with measuring and evaluating the results during full-scale tests arise.

\section{Conclusion}

This paper presents a number of results obtained in the development and testing of virtual benches and digital platforms built on the basis of the digital twin of the designed product of the automotive industry. It is shown that the described software package can significantly speed up not only the vehicle design process, but also reduce the cost of testing it. Using the example of virtual benches for crash tests, it is shown that the developed digital platform allows assessing passive safety for vehicles in the non-commercial and commercial sector. 
In the development of such platforms and benches, the adequacy of the applied mathematical model, as well as the methods of computer simulation, plays a key role. As a rule, it is crucial how the finite element models are implemented, and what physics is chosen for this or that digital twin and virtual bench.

It is obvious that the proposed testing approach based on a simulation model or a digital twin makes it possible to automate such important processes as optimization of the topology of mechanical structures, parameters of body elements, and vehicle pillars. For optimization, a gradient method or artificial intelligence technologies, genetic algorithms can be applied.

Similar virtual benches can be obtained for assessing the electronic and power systems of the designed product, the comfort of passengers and the driver, water resistance of the body and visibility, given the design features of the product.

The digital platform, as a set of software for design, verification and assessment, also allows the implementation of certification programs for designed products. It can be a separate direction in the development of digital platforms and their basic elements, i.e. virtual benches and digital twins of the designed products of the automotive industry. Thus, it can also be used for the products of the aircraft building, shipbuilding industries, or for the objects of railway transport in terms of transport systems.

\section{References}

1. D. Kim, H. Peng, S. Bai, J. Maguire, IEEE Transactions on Control System Technology 15(3), 474-482 (2007)

2. B. Dang, A. Zhilenkov, AIP Conf. Proc. 2034, 020007 (2018) https://doi.org/10.1063/1.5067350

3. A.I. Borovkov, L.B. Maslov, K.S. Ivanov, E.N. Kovaleva, F.D. Tarasenko, M.A. Zhmaylo, IOP Conf. Ser.: Mater. Sci. Eng. 986, 012034 (2020)

4. A.A. Zhilenkov, D. Denk, IEEE Conference of Russian Young Researchers in Electrical and Electronic Engineering (EIConRus) $1104 \quad$ (2017) doi: 10.1109/EIConRus.2017.7910748

5. D. Vuksanović, J. Ugarak, D. Korčok, Sinteza 2016 - International Scientific Conference on ICT and E-Business Related Research, 293-298 (2016)

6. A.A. Zhilenkov, I.R. Epifantsev, IEEE Conference of Russian Young Researchers in Electrical and Electronic Engineering (EIConRus) 1032 (2018) doi: 10.1109/EIConRus.2018.8317265

7. Z. Chen, S. Cao, Z. Mao, Energies 11, 28 (2018)

8. A.A. Zhilenkov, IEEE Conference of Russian Young Researchers in Electrical and Electronic Engineering (EIConRus) 400 (2018) doi: 10.1109/EIConRus.2018.8317117

9. D.O. Solovev et al, IOP Conf. Ser.: Mater. Sci. Eng. 747, 012076 (2020) 